\title{
Shaken not stirred: On Permanence in Ecological Communities
}

Vincent A.A. Jansen (vincent@lyapunov.zoo.ox.ac.uk) Karl Sigmund (ksigmund@esi.ac.at)

\section{Approved by Ulf Dieckmann (dieckman@iiasa.ac.at) Project Coordinator, Adaptive Dynamics Network}




\section{Contents}

1 A kind of stability 1

2 Community construction $\quad 3$ 


\title{
About the Authors
}

\author{
Vincent A.A. Jansen \\ Department of Zoology \\ University of Oxford \\ South Parks Road \\ Oxford OX1 3PS, U.K. \\ Karl Sigmund \\ Institut für Mathematik \\ Universität Wien \\ Strudlhofgasse 4 \\ A-1090 Vienna, Austria \\ and \\ Adaptive Dynamics Network \\ International Institute for Applied Systems Analysis \\ A-2361 Laxenburg, Austria
}

\section{Acknowledgment}

We want to thank J. Hofbauer, U. Dieckmann, S. Schreiber and R. Law for helpful discussions. 


\title{
Shaken not stirred: On Permanence in Ecological Communities
}

\author{
Vincent A.A. Jansen \\ Karl Sigmund
}

\section{A kind of stability}

All models of ecological communities are approximations: it would be pointless to burden them with too many contingencies and details. On the other hand, they would be of little help if they were not robust against the kind of perturbations and shocks to which a real ecosystem is ceaselessly exposed. They have to exhibit some kind of stability. But which kind? For a considerable time, it used to be understood that stability meant local stability of an equilibrium, i.e. asymptotic stability in the sense of Lyapunov: any sufficiently small perturbation is promptly offset by a small countermove back to the stationary state. But this notion, which was developed by physicists and engineers and serves perfectly well to describe the stability of a control mechanism or a mixture of chemicals, is not really appropriate for ecologists brought up on lynx-hare cycles and the antics of budworm populations. Environmental perturbations are often vigourous shake-ups, rather than gentle stirrings. Furthermore, there may be no stable equilibrium to return to, but a periodic or chaotic attractor.

Lewontin (1969) wrote in his paper on The meaning of stability that 'the presence or absence of species is sometimes the point of interest regardless of some variation in their numbers'. And indeed an ecological community is often simply described by its cast of species - in mathematical terms, by the support of the vector $\mathbf{x}=\left(\mathbf{x}_{1}, \ldots, \mathbf{x}_{\mathbf{n}}\right)$ of the population densities, i.e. by those species $i$ for which $x_{i}>0$. Of course, small values of $x_{i}$ render extinction very likely. Such small values are obviously unavoidable in the initial phase of invasion. But after a transient phase, the dynamics should never return to such dangerously small values.

Let us formulate this in mathematical terms. We consider dynamical systems on the positive orthant $R_{+}^{n}$ which have the property that they leave the boundary faces invariant: whenever a species $i$ is absent (i.e. $x_{i}=0$ ), it remains absent forever. This is to model ecological systems where new species can only be introduced by external causes (speciation, or immigration). For simplicity of exposition, we shall only consider systems given by ordinary differential equations. (In the survey by Hutson and Schmitt (1992), a wide variety of other dynamics is also considered). Such a system is said to be permanent if the boundary (including the points at infinity) is a repeller: more precisely, if there exists a compact set $K$ in the interior of $R_{+}^{n}$ such that every orbit starting at a point $\mathbf{x}$ in the interior satisfies $\mathbf{x}(t) \in K$ if $t$ is sufficiently large, or in other words if there exist constants $0<a<A$ such that 
whenever $x_{i}(0)>0$ for $i=1, \ldots, n$ then

$$
a<\liminf x_{i}(t) \leq \lim \sup x_{i}(t)<A
$$

for all $i$. This notion of permanence, which was first introduced by Schuster et al. (1979), is also known under different aliases, like permanent coexistence or uniform persistence.

Permanence is a stronger notion than persistence, which requires that the $\omega$ limit of every orbit in the interior is bounded away from the boundary (i.e. that $\liminf x_{i}(t)>0$ for all $i$ ). An example of a model which is persistent, but not permanent is given by the familiar Lotka-Volterra predator-prey equation without self-limitation, i.e. $\dot{x}_{1}=x_{1}\left(a-b x_{2}\right), \dot{x}_{2}=x_{2}\left(-c+d x_{1}\right)$. Although the interior of the positive orthant $R_{+}^{2}$ is filled with periodic orbits surrounding the equilibrium $(c / d, a / b)$, this system is not permanent: the boundary is not repelling, since there exist periodic orbits (and hence $\omega$-limits) arbitrarily close to it. To see why this matters, it is instructive to perturb this model by adding some external noise; the orbit will 'drift' and sooner or later densities will be reached for which extinction is certain. For a classification of the different persistence and permanence notions, we refer to Freedman and Moson (1982).

A simple argument based on Brouwer's fixed point theorem shows that a permanent dynamical system must always have a fixed point in the interior of $R_{+}^{n}$; but this point need not be locally stable. No fixed point on the boundary of $R_{+}^{n}$ can be a limit point of an interior orbit. Generically, this implies that a fixed point on the boundary cannot be saturated: there exists at least one $i$ such that $x_{i}=0$ but the growth rate of $x_{i}$ is positive in a neighborhood of this equilibrium. This means that some species which is missing can invade at this equilibrium. Neither the existence of an interior equilibrium nor the absence of saturated equilibria on the boundary is sufficient for permanence.

Permanence shares two conceptual weaknesses with local stability. One is that the size of 'allowable' perturbations is not specified: just as a locally stable fixed point can be very close to the boundary of its basin of attraction, so the interior attractor of a permanent system can be very close to the boundary face of the positive orthant. The other weakness is the lack of structural stability: just as some dynamical systems with a locally stable equilibrium can be approximated by systems without a locally stable equilibrium, so there exist permanent systems such that ecological equations which are arbitrarily close are not permanent. We conjecture however that generically, a permanent system is robustly permanent, in the sense that every small perturbation is also permanent.

The most useful sufficient condition for permanence is the existence of an average Lyapunov function (Hofbauer, 1981, Hutson, 1984). This is a function vanishing on the boundary and positive on the interior which grows along every interior orbit sufficiently close to the boundary, or - to ask for less - whose time average grows, so that if one waits for a sufficiently long time, one can be sure to be further away from the boundary than one was before. In the context of a Lotka-Volterra equation

$$
\dot{x}_{i}=x_{i}\left(r_{i}+\sum a_{i j} x_{j}\right)
$$

this reduces to a system of inequalities (Jansen, 1987): if there exists a $\mathbf{p}=$ 
$\left(p_{1}, \ldots, p_{n}\right)$ with $p_{i}>0$ for all $i$ such that for every equilibrium $\mathbf{z}$ on the boundary,

$$
\sum p_{i}\left(r_{i}+\sum a_{i j} z_{j}\right)>0
$$

(a conditions that can easily be checked by linear programming), the system is permanent. The condition is not necessary for permanence if $n>3$. But if the Lotka-Volterra equation is permanent, then there is a unique equilibrium $\hat{\mathbf{x}}$ with all species present, and it is the limit of all time-averages of orbits in the interior of the state-space. If $D$ is the Jacobian at $\hat{\mathbf{x}}$, then $(-1)^{n} \operatorname{det} D>0$, and trace $D<0$. Furthermore, $(-1)^{n} \operatorname{det} A>0$, where $A$ is the matrix of the interaction terms $a_{i j}$; and if there exists an equilibrium in the interior of the face $x_{k}=0$, then the determinant of the matrix obtained by deleting the $k$-th row and column from $A$ has the sign of $(-1)^{n-1}$. For an extensive account of permanence in Lotka-Volterra systems, we refer to Hofbauer and Sigmund (1998).

These conditions can lead to simple criteria for permanence in low-dimensional systems (see Butler, Freedman and Waltman, 1983, Hutson and Vickers, 1983, Kirlinger 1984). They often boil down to the question whether heteroclinic cycles on the boundary are repelling or not. Heteroclinic cycles consist of saddle equilibria $E_{1}, \ldots, E_{k}$ connected by orbits: $o_{i}$ leads from $E_{i}$ to $E_{i+1}$, in the sense that its $\alpha$-limit is $E_{i}$ and its $\omega$-limit $E_{i+1}$, and the indices are counted modulo $k$. Such cycles are chain-recurrent, and hence possible candidates for $\omega$-limits of interior orbits (Garay, 1987 and 1992).

Consider, for instance, a system with two self-limiting prey species and two predator species. If the prey are in bistable competition, it could happen that an equilibrium with prey 1 only is invaded by predator 1 . The resulting equilibrium between the two species can in turn be invaded by prey 2, which eliminates the two other species. But then, predator 2 can invade, and the resulting equilibrium can be invaded by prey 1 , which eliminates prey 2 and predator 2 , etc... (similar cycles are quite likely to occur if two host genotypes are attacked by two strains of parasites, each specialised on a different genotype). The result can either be a permanent coexistence of the four species (not necessarily in equilibrium) or an endless cycle of extinctions and re-invasions (see Kirlinger, 1989).

\section{Community construction}

Permanence has proved a powerful tool for the study of ecosystems. For instance, the keystone role played by a top predator can be studied by these means. One might think that an ecological network will benefit from the removal of an exploiter sitting at the top of the food chain. But this is not necessarily so. It can happen, for instance, that two prey and one predatory species form a permanent system, but that the two prey species, in the absence of the predator, are no longer permanent: one of them eliminates the other. In this sense, a predator can 'stabilise' (i.e. mediate coexistence of) a two-prey system which, by itself, is not permanent because one species dominates the other. On the other hand, a bistable system of two competing species cannot be stabilised by the introduction of a suitable predator. But it can be stabilised by the introduction of two predatory species, each one specialised upon one 


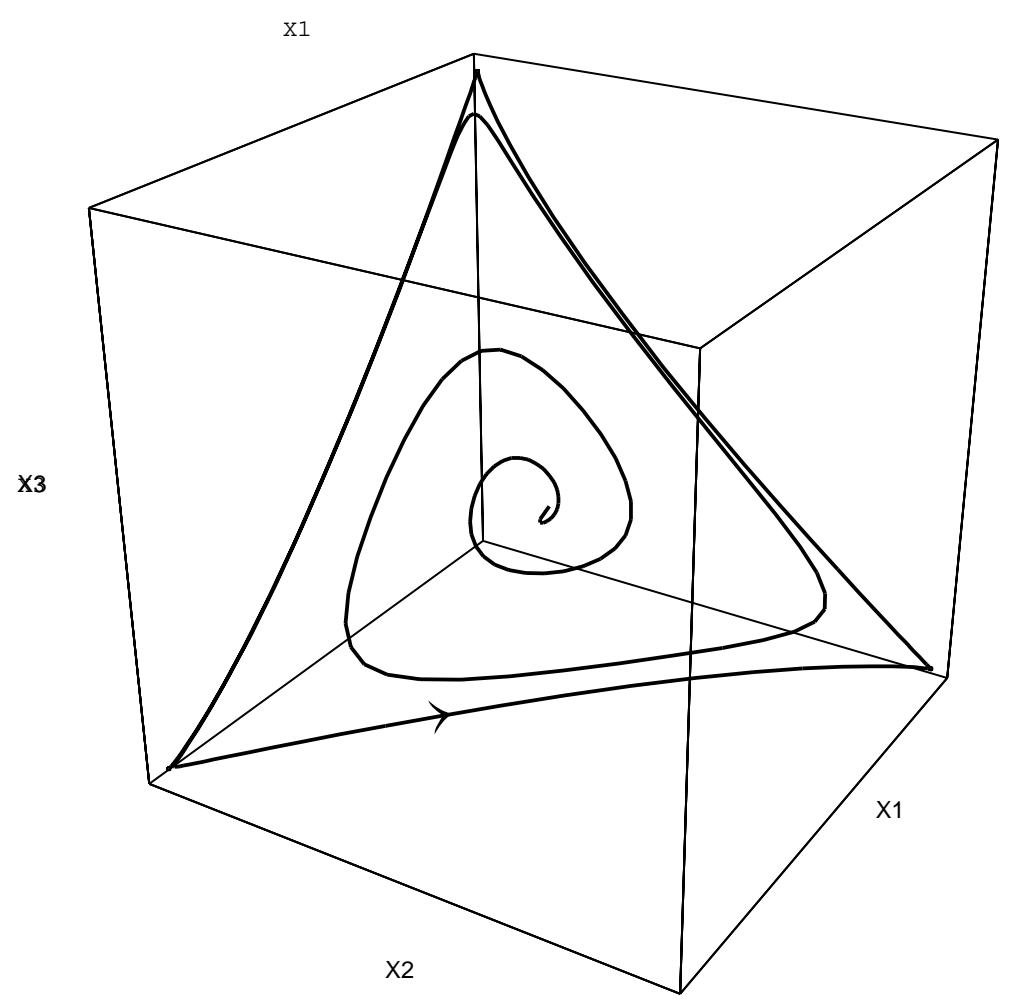

Figure 1: A heteroclinic attractor for a Lotka-Volterra model with three competing species in a rock-scissors-paper cycle. The orbit approaches the boundary.

of the prey. Alternatively, it can be stabilised by the introduction of one competing species and one predatory species. Similarly, a non-permanent system consisting of three competing species forming a rock-scissors-paper cycle (see Fig.1 for an example due to May and Leonard, 1975) can be rendered permanent by the mediation of an additional competing species, or by a predatory species with suitable parameters. It follows that such a predator is a key-stone component of the ecosystem.

These results assumed Lotka-Volterra interactions, but recently, Schreiber (1997) has studied the possibilty of permanence-mediating predators between bistable prey in a considerably broader context. He also adressed higher-dimensional models, and showed that in a community of $n$ prey species which are founder-controlled (i.e. whichever species is the first to establish itself prevents all others to invade), it takes $n$ specialist predators to mediate permanence (Schreiber, 1998). There exists a heteroclinic network on the boundary of the full system of $n$ preys and $n$ predators. Removal of any predator leads to the collapse of the entire system: only one prey remains. In Schreiber's terms, this is like a delicately balanced ecological wheel whose spokes are the predator-prey linkages. Removal of one spoke results in the collapse of the wheel.

There exists a wealth of theoretical and empirical results on trophic levels, connectance, complexity of the dynamics, resistance to invasion etc... (see Yodzis, 1990, Pimm, Lawton and Cohen, 1991). In order to understand the structure of multi-species communities, theoretical ecologists turn increasingly to the method of 
community construction. Essentially, the community assembly technique consists in devising a hypothetical pool of species and then setting up an artificial ecosystem by sequential invasions. Some of the invaders will be eliminated, others can grow and get a foothold in the population, possibly eliminating some other species. The earlier studies were often too focussed on locally stable equilibria, and missed a substantial amount of possible 'succession chronicles'. The permanence notion is more appropriate (for a fair appraisal, see Morton et al, 1996). The initial invasion process can be well described by certain Lyapunov exponents, even if the attractor of the resident system is a heteroclinic cycle, but it can be difficult to predict the ultimate success of the invader (see Hofbauer, 1998).

The extensive work by Law and Blackford (1992) and Law and Morton (1993, 1998) helps in understanding the role of history in community assembly. Numerical evidence suggest that the resistance to a further invasion increases with time, at least on average. Frequently, the succession of permanent communities leads to an end point which cannot be invaded by a further species. This end state may depend on the (contingent) sequence of arrivals, but in general, the number of alternative end points is small. No such end point can be a subset of another end point, by the way. It can also happen that there is no end point at all. In this case, the community structure is transient: some species undergo endless cycles of elimination and successful re-invasion. Interestingly, end points often lack re-assembly paths; they cannot be obtained through a succession of stepwise increasing permanent subcommunities, but need catalytic species which eventually go extinct. In order to understand how the community was assembled, one needs to know more than just the species which make it up.

The assumption that species invade one at a time is the very opposite from the assumption that the ecological community is assembled at one stroke, and yields very different results concerning permanence, resistance to invasion, connectivity etc. One could also study intermediate scenarios, where the resident community is invaded by several species at the same time. A framework for this approach is the community transition graph introduced in Schreiber (1998). Multiple invasions occur, for instance, if two areas are separated by a barrier that is difficult but not impossible to cross, like a mountain range or a fordable river. This can also be modelled by diffusion, and opens up another field of investigations (see Levin, 1974, Takeuchi, 1996). For instance, Hastings (1978) has shown that if patches are governed by the same Lotka-Volterra dynamics (2) leading to a strong form of global stability, and if dispersal is symmetric, then the $n$-patch model is globally stable. If the $r_{i}$ are different in the two patches, global stability need no longer hold (Hofbauer et al, 1996).

Can a system of diffusively coupled 'patches' be permanent while the same system without diffusion is not? If all patches are governed by the same differential equations, the $n$-patch system cannot be made permanent. Indeed, if the species' densities are equal in every patch, and if there is no cost associated with moving, the net effect of movement on the local densities is nil and the spatial system behaves just like an isolated patch: the boundary, therefore, is not repelling.

On the other hand, a spatially heterogeneous system can be made permanent by diffusion. One example is a system of two species, specialised on types of food which 
are only available in different parts of the habitat. Diffusion can obviously make this system permanent (Cantrell et al, 1996). The following example is less obvious: consider a system of two different patches, in one of which the dynamics are governed by the Lotka-Volterra model for prey-predator interaction without self limitation. Prey and predators can move into the other patch, a rather barren refuge where they cannot feed, be eaten or reproduce; they can only die or move back. Obviously neither patch is permanent when isolated. The system has been studied with various ecological interpretations (Holt 1985, Weisser and Hassell 1996). It has a globally stable equilibrium and is therefore permanent (Weisser et al. 1997). One might wonder what happens if the refuge is not completely barren. If prey and predator can grow and interact in the other patch we obtain a system of two coupled LotkaVolterra patches. When prey or predator does not move, the equilibrium can be shown to be globally stable. When both species can move without cost, numerical results show that the equilibrium is stable (Nisbet et al. 1992), but it seems as yet unclear whether the system is permanent.

When the Lyapunov function for diffusive Lotka-Volterra systems is applied to two identical Lotka-Volterra patches with predator and prey populations, it can be shown that every orbit starting in the interior will converge to the diagonal - that subset of the state space where the densities of each species are equal in the two patches. Both patches will exhibit the same dynamics as an isolated patch, with periodic orbits arbitrarily close to the boundary.

This could lead to the conclusion that for systems of identical patches and diffusion rates, space does not matter. Surprisingly, this is not always true. For the spatial variant of the model the larger closed orbits are unstable for a suitable choice of parameters (Jansen 1994, 1995; Jansen and De Roos 1998). For initial conditions close to an unstable closed orbit on the diagonal space, the solution will initially be repelled from the diagonal and later converge to a smaller closed orbit on the diagonal. Numerical simulations show that solutions move away from the boundary while the amplitude of the fluctuation is decreased (Fig. 2 ).

Although the spatial system is not permanent, the situation is comparable to examples in Butler and Waltman (1981), where the set of initial conditions that lead to the boundary has measure zero. It is easy to define a corresponding notion of 'almost permanence' (we have only to require that (1) holds for almost all interior orbits), but it seems difficult to find workable criteria for almost permanence which depend only on what happens near the boundary. Such techniques may eventually shed light on a conjecture by Nicholson and Bailey (1935) who, after having observed that the densities of host and parasites in their model fluctuate with ever increasing amplitudes, wrote: "A probable ultimate effect of increasing oscillations is the breaking up of the species-population into numerous small, widely separated groups which wax and wane and then disappear, to be replaced by new groups in previously occupied situations". The spatial Nicholson-Bailey model can indeed give rise to a spatial pattern, but this is not enough to ensure permanence, because another attractor can exist simultaneously, on which the predator drives the prey to extinction just as in the non-spatial model (Hassell et al, 1991: Adler, 1993).

The concept of species that persist through a spatial pattern is related to that of a fugitive species: populations that would be doomed to extinction when confined to 


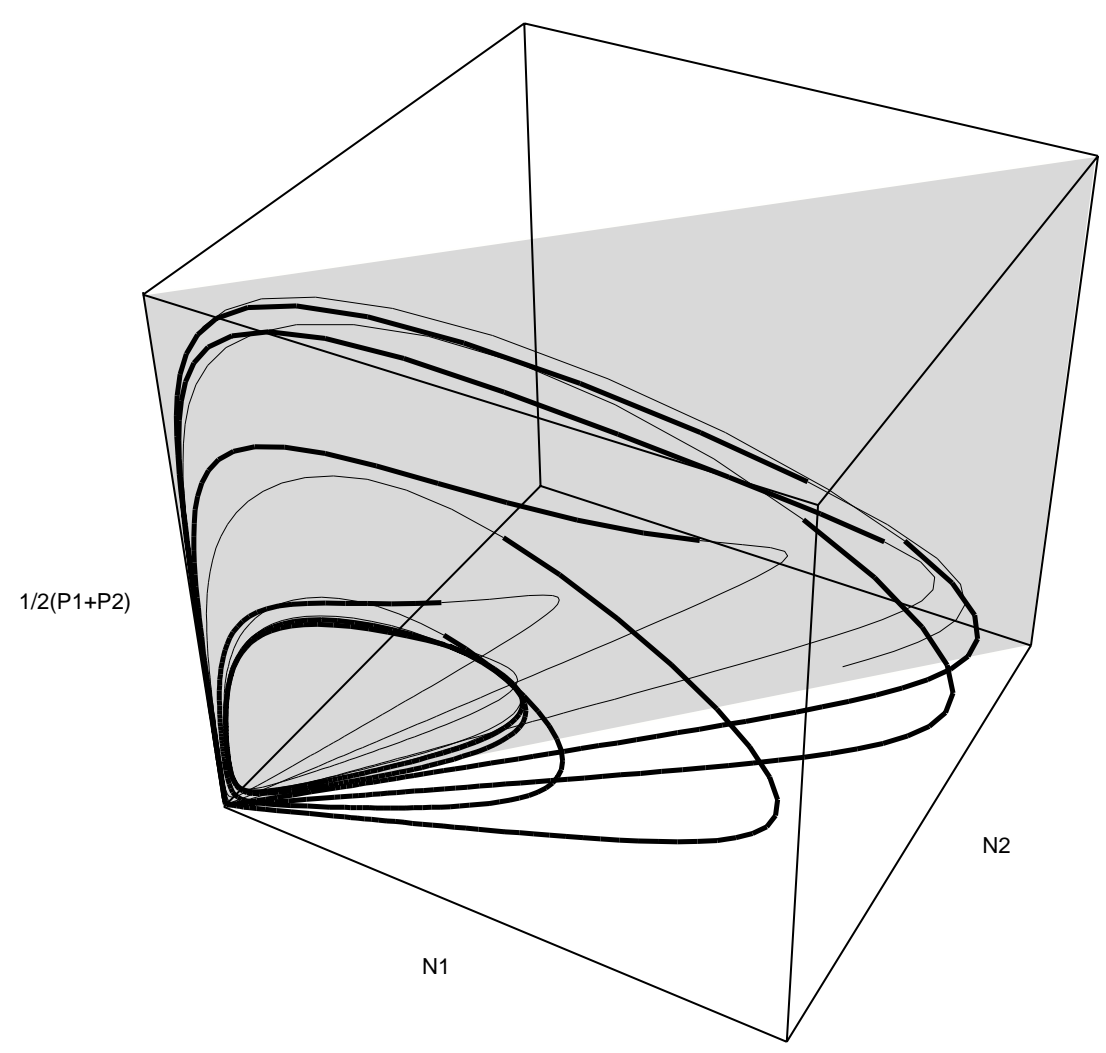

Figure 2: A Lotka-Volterra predator-prey model with two patches coupled by predator migration. N1 and N2 are the prey densities in the two patches, P1 and P2 the predator densities - their mean is shown on the vertical axis. The shaded area corresponds to the diagonal $N 1=N 2$, the bold part of the orbit is in the half-space $N 1>N 2$. The orbit starts far out of equilibrium, but close to the diagonal. After a transient phase, the difference between the two patches decreases, but now the oscillations of the densities are much smaller. Under added noise the dynamics of the spatial model is very different from the non-spatial model. 
a single habitat but can survive by constantly moving between habitats. A system of patches in which the quality of the patches varies over time can be permanent, even if one such patch is not. The simplest examples are discrete time models for the allocation of offspring over two patches. Let us assume that one of these patches is a sink in which the reproductive success of a population constrained to this patch is less than one, and the reproductive success if constrained to the other patch is exactly one. (Due to the fluctuations, reproductive success is given by the limit of the geometric mean of the growth rates). The reproductive success of individuals who distribute their offspring over the two patches can exceed one (Yoshimura and Jansen, 1996; Roughgarden, 1998); allocating offspring to a sink habitat can therefore be evolutionarily stable (Holt, 1997). Even when both habitats are sinks, and hence non-permanent, the geometric mean reproductive success in the combined system can exceed unity. In this way a system with two habitats can be permanent while the same system would not be permanent if either of the habitats were removed (Jansen and Yoshimura, 1998). Although this provides a possible mechanism by which fugitive species can survive, many questions remain unanswered. In particular it would be interesting to know how 'fugitive' a species must be in order to survive. This question is easy to answer for the above described offspring allocation models, but for more realistic metapopulation models this is largely unsolved.

\section{References}

Adler, F.N. (1993) Migration alone can produce persistence of host-parasitoid models. Am. Nat. 141, 642-650.

Butler, G. and Waltman, P. (1981) Bifurcation from a limit cycle in a two predatorone prey ecosystem modelled on a chemostat J. Math. Biol. 12, 295-310.

Butler, G., Freedman, H. and Waltman, P. (1986) Uniformly persistent systems, Proc. Amer. Math. Soc. 96, 425-430.

Cantrell, R.S., Cosner, C. and Hutson, V. (1996) Ecological models, permanence, and spatial heterogeneity, Rocky Mt. J. Math. 26, 1-35.

Freedman, H. and Moson, P. (1990) Persistence definitions and their connections, Proc. Amer. Math. Soc. 109, 1025-33.

Garay, B.M. (1989), Uniform persistence and chain recurrence, J. Math. Anal. Appl. 139, 372-381.

Garay, B.M. (1992) Chain recurrent subsets of $\partial R_{+}^{p}$ as $\omega$-limit sets. Comment. Math. Univ. Sr. Pauli 41, 23-34.

Hassell, M.P., Comins, H. and May, R.M. (1991) Spatial structures and chaos in insect population dynamics, Nature 353, 252-258.

Hastings, A. (1978) Global stability in Lotka-Volterra systems with diffusion, J. Math. Biol. 6, 163-168.

Hastings, A. (1988) Food web theory and stability', Ecology 69, 1665-1668. 
Hofbauer, J. (1981) A general cooperation theorem for hypercycles, Monatshefte Math. 91, 233-240.

Hofbauer, J. (1998) Invasion, permanence and heteroclinic cycles, to appear.

Hofbauer, J. and Sigmund, K. (1989) On the stabilising effect of predators and competitors on ecological communities, J. Math. Biol. 27, 537-548.

Hofbauer, J. So, J.W.H. and Takeuchi, Y. (1996) Global stability of competition in a spatially heterogeneous environment, Diff. Equs. and Dyn. Syst. 4, 213-23.

Hofbauer, J. and Sigmund, K. (1998) Evolutionary games and population dynamics, Cambridge UP.

Hutson, V. and Schmitt, K. (1992) Permanence and the dynamics of biological systems, Math. Biosci. 111, 1-71.

Hutson, V. (1984) A theorem on average Ljapunov functions, Monatshefte Math. 98, 267-275.

Hutson, V. and Vickers, G. (1983) A criterion for permanent coexistence of species, with an application to a two-prey, one-predator system, Math. Biosci. 63, 253-269

Holt, R.D. (1985) Population dynamics in two patch environments: some anomalous consequences of an optimal habitat distribution, Theor. Pop. Biol. 28, 181208.

Holt, R.D. (1997) On the evolutionary stability of sink populations, Evol. Ecol. 11, 723-731.

Jansen, V.A.A. and de Roos, A.M. (1998) Predator-prey dynamics across spatial scales: the role of space in reducing population cycles. In: The Geometry of Ecological Interactions: Simplifying spatial complexity. (U. Dieckmann, R. Law and J.A.J Metz, eds.) Cambridge UP.

Jansen, V.A.A. and Yoshimura, J. (1998) Populations can persist in environments consisting of sink habitats only, Proc. Natl. Acad. Sci 95, 3696-8.

Jansen, V.A.A. (1994) Theoretical Aspects of Metapopulation Dynamics. PhD thesis, Leiden University.

Jansen, V.A.A. (1995) Regulation of predator-prey systems through spatial interactions: a possible solution to the paradox of enrichment. Oikos 74, 384-390.

Jansen, W. (1987) A permanence theorem for replicator and Lotka-Volterra systems, J. Math. Biol. 25, 411-422.

Kirlinger, G. (1989) Two predators feeding on two prey species: a result on permanence, Math. Biosci. 96, 1-32.

Lewontin, R. (1969) The meaning of stability. Brookhaven Symp. Biol. 22, pp. 13-24.

Law, R. and Blackford, J.C. (1992) Self-assembling food webs: a global viewpoint of co-existence of species in Lotka-Volterra communities, Ecology 73, 567-578.

Law, R. and Morton, R.D. (1993) Alternative permanent states of ecological communities, Ecology 74, 1347-1361. 
Law, R. and Morton, R.D. (1998) Permanence and the assembly of ecological communities, Ecology, in press.

Levin, S.A. (1974) Dispersion and population interactions, Amer. Nat. 108, 207228.

May, R.M. and Leonard, W.J. (1975) Nonlinear aspects of competition between three species, SIAM J. Appl. Math. 29, 243-253.

Morton, R.D., Law, R. Pimm, S.L. and Drake, J.A. (1995) On models for assembling ecological communities, Oikos 75, 493-9.

Nicholson, A.J. and Bailey, V.A. (1935) The balance of animal populations, Proc. Roy. Soc. London 3, 551-598.

Nisbet, R.M., Briggs, C.J., Gurney, W.S.C., Murdoch, W.W. and Stewart-Oaten, A. (1992) Two-patch metapopulation dynamics. In: Patch dynamics in terrestial, freshwater and marine ecosystems. Lect. Notes Biomath 96, Springer, Berlin.

Nee, S. (1990) Community construction, Trends in Ecology and Evolution 5, 337340.

Pimm, S.L., Lawton, J.H. and Cohen, J.E. (1991) Food web patterns and their consequences, Nature 350, 669-674.

Roughgarden, J. (1998) A primer for ecological theory, Prentice hall, New Jersey.

Sabelis, M.W. and Diekmann, O. (1988) Overall population stability despite local extinction, Theor. Pop. Biol. 34, 169-176.

Schreiber, S. (1997) Generalists and specialist predators that mediate permanence in ecological communities, J. Math. Biol. 36, 133-48.

Schreiber, S. (1998) On the stabilizing effect of predators on founder-controlled communities, to appear in Canad. Appl. Math. Quarterly.

Schreiber, S. (1998) Successional stability of vector fields in dimension three, to appear in Proc. AMS.

Schuster, P., Sigmund, K. and Wolff, R. (1979) Dynamical systems under constant organisation III: Cooperative and competitive behaviour of hypercycles, $J$. Diff. Equs. 32, 357-368.

Takeuchi, Y. (1996) Global dynamical properties of Lotka-Volterra systems, World Scientific, Singapore.

Weisser, W.W. and Hassell, M.P. (1996) Animals 'on the move' stabilize hostparasitoid systems. Proc. Roy. Soc. London B 263, 749-754.

Weisser, W.W., Jansen, V.A.A. and Hassell, M.P. (1996) The effects of a pool of dispersers on host-parasitoid systems, Proc. Roy. Soc. London B 263, 749754.

Yodzis, P. (1990) Introduction to theoretical ecology, Harper and Row, New York.

Yoshimura, J. and Jansen, V.A.A. (1996) Evolution and population dynamics in stochastic environments Res. Pop. Ecol. 38, 165-182. 